\title{
Solvation Dynamics in Water: 4. On the Initial Regime of Solvation Relaxation
}

\author{
Rossend Rey* \\ Departament de Física, Universitat Politècnica de Catalunya, Campus Nord B4-B5, Barcelona 08034, Spain. \\ James T. Hynes ${ }^{\dagger}$ \\ PASTEUR, Department of Chemistry, École Normale Supérieure, \\ PSL University, Sorbonne Université, CNRS, 75005 Paris, France \\ Department of Chemistry, University of Colorado, Boulder, CO 80309 USA
}

(Dated: August 6, 2020)

\begin{abstract}
It is shown, by means of numerical and analytic work, that initial molecular momenta play little significant role in the initial fast solvation relaxation that follows electronic excitation of, and charge creation for, a standard model system of a solute in water. Instead, the nonequilibrium dynamics are predominantly described by non-inertial "steering" by the torques directly generated by the newly created charge distribution. It is this process that largely overcomes inertia, and drives the relaxation dynamics on a time scale of a few tens of femtoseconds in the key initial regime of the dynamics. These results are discussed in the context of commonly employed descriptions such as inertial, Gaussian, and underdamped dynamical behavior.
\end{abstract}

\section{INTRODUCTION}

The channeling of energy in chemical reactions depends critically on translational, rotational and vibrational modes of the solvent. One stepping stone towards a fuller understanding - since it provides access to the time scales of solvent dynamics - comes from the analysis of solvation relaxation, i.e. the simpler process by which the solvent adjusts to an electronic excitation and accompanying sudden charge distribution change of a solute molecule within it. This topic has accordingly attracted considerable experimental, theoretical and computational attention over the years, ${ }^{1-31}$ continuing to the present, ${ }^{32-53}$ including explorations for increasingly complex systems such as ion transport, ${ }^{54,55}$ supercritical water, ${ }^{56-58}$ ionic liquids, ${ }^{59-61}$ proteins and biomolecules, ${ }^{62-75}$ inorganic complexes, ${ }^{76-78}$ and active matter $^{79}$ (the literature potentially citable in this sentence is very extensive, our referencing-while fairly extended - is of necessity more limited). The excitation induces an ensuing solute frequency red shifting that encapsulates the generally complex dynamics that accomplishes the free energy minimization as the solvent establishes equilibrium with the new charge distribution. This time-dependent shift has constituted the basic observable over which the study of the solvation process has pivoted. In this connection, it is standard practice to focus, both experimentally and theoretically, on the normalized dynamic frequency shift (or Stokes shift) function (FSF)

$$
S(t) \equiv \frac{\omega(t)-\omega(\infty)}{\omega(0)-\omega(\infty)},
$$

with $\omega(t)$ denoting the time-dependent fluorescence emission frequency, which reflects the energy gap $\Delta E$ between the excited and ground electronic states.

A striking fundamental feature of $S(t)$, first found in numerical simulation (most often via a certain time correlation function approximation for $S(t)$, to be discussed within $^{9-19}$ ), is its bimodal character: a fast subpicosecond initial decay, followed by a slow component in the picosecond range. Experimental verification of this bimodal character came with the work of Jimenez et al. ${ }^{20}$ on excitation of the coumarin dye in water, where an initial dominant decay with a time scale under 50 fs was reported.

Such bimodality has been captured in simulations by the idealized model of a neutral monatomic solute which instantaneously acquires a positive/negative unit charge in a solvent - often water, the solvent of choice in this article. This capture helps to explain the prominent role that the model has played in theoretical studies, particularly for a modeled water solvent. ${ }^{10,21-24,32-34,55,58,80,81}$ Figure 1 displays the FSF for a water solvent obtained for this model from a nonequilibrium dynamics simulation. ${ }^{32}$ This exhibits an initial dominant fast decay within a time of approximately $20 \mathrm{fs}$, followed by a slow component that lasts up to 1-2 ps (with an oscillatory transient in its beginning stages). We term the former fast component the "initial" regime behavior throughout this article.

Since by itself the FSF (either experimental or simulated) provides only indirect - as opposed to detailed molecular level - information on solvation dynamics, numerical simulation has been indispensable for its theoretical interpretation. The usual approach has relied on the calculation of related equilibrium time correlation functions (TCFs), which are an approximation to a (much less common) direct nonequilibrium dynamics approach. But even with these techniques in hand, there have been, and remain, a number of issues left unresolved concerning solvation dynamics, especially regarding the initial dynamics. This is of particular concern for at least two reasons: first, because these dynamics can often constitute a significant fraction of the solvent relaxation, and second, this initial regime is often key for the solvent dynamical influence on chemical reaction rates. ${ }^{8,12,26,28,53,82-94}$

We will focus in this article on the issue of the initial time regime of solvation dynamics - and that for a 


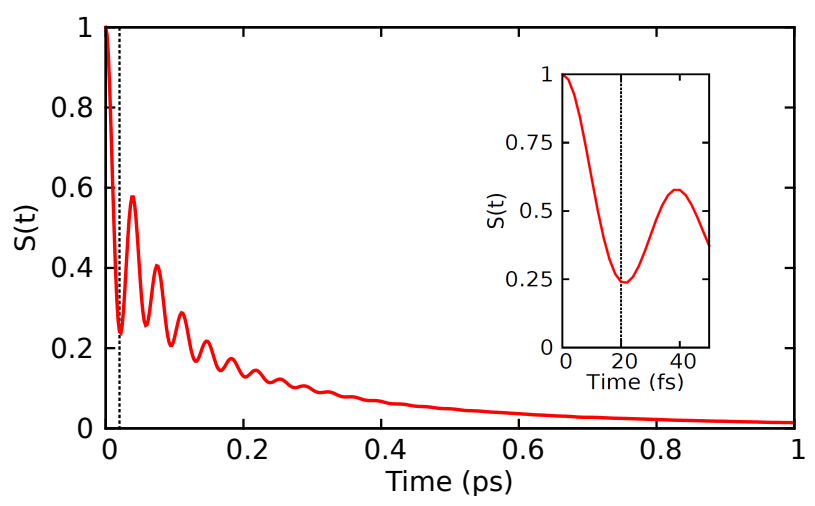

FIG. 1: Nonequilibrium dynamics simulation result ${ }^{32}$ for the FSF function $S(t)$, using Eq. 2, for a neutral solute (similar to an oxygen atom in size; cf Sec. II) in rigid water solvent acquiring a positive unit charge (see Sec. II for a description of the solute, solvent and methodology). The vertical dashed line marks a time of $20 \mathrm{fs}$; this defines the "initial" regime, a principal focus of this article, the insert highlights this region. This $S(t)$ result is very similar to the original NED result on a similar system. ${ }^{10}$ The corresponding result for an initially created negative charge is presented in the SI (Sec. S1, with further details given in S2).

particular solute/water model - but even with that limitation, a perusal of the literature reveals very frequent conflicting interpretations for the characterization of this initial regime; disparate adjectives employed include inertial, Gaussian, and underdamped, sometimes with no accompanying restriction to any particular solute or solvent type, or with conflicting adjectives applied to the initial regime for the same solvent.

For the present contribution, we will henceforth adopt the specific definitions ${ }^{13}$ that: "inertial" means free streaming solvent dynamics determined by the initial momenta rather than by charge change-induced forces and/or torques (i.e. it is the initial unforced free streaming motion changing particle positions and thus the energy gap $\Delta E),{ }^{13-15,95}$ that "Gaussian" behavior refers specifically to initial Gaussian time dependence for nonequilibrium or (approximate) equilibrium measures of the solvation dynamics function $S(t),{ }^{12-15,90,91,96,97}$ (rather than more general issues of Gaussian statistics ${ }^{13,41,84}$ ) and that "underdamped" means that the solvent motion depends critically upon forces and/or torques, typically exhibiting decaying oscillations, and is to be contrasted with "overdamped".

In this effort, we will pursue the nonequilibrium dynamics (NED) approach to solvation dynamics, in an attempt to clarify a number of the issues associated with the three descriptions indicated above. Our choice of NED is due to its character as a direct approach that most closely (though certainly not perfectly) relates to experiments, and avoids the inevitable approximations necessary to achieve a TCF description; we do not however enter into any general discussion of the "linear response theory" issues concerning the disparities between
NED and TCF predictions. ${ }^{10,12-17,39,40,45,81}$

Within the NED approach, we recently ${ }^{32-35}$ presented an alternative perspective for solvation dynamics founded on the computation of the nonequilibrium energy fluxes ensuing as a result of the initial excitation. This perspective exploited a power/work energy flow methodology previously implemented for vibrational/rotational relaxation. ${ }^{36-38}$ This methodology's capital merit rests on its ability to provide explicit and quantitative information about the participation of each solvent molecule and that molecule's different types of motion: vibrational, rotational and translational. The relevance for the FSF solvation dynamics problem is the realization that the relaxation can be regarded as ultimately an electronic to solvent molecule energy phenomenon. In the energy flow perspective's first application to the solvation dynamics problem, its capabilities were exploited ${ }^{32-35}$ for the standard monoatomic neutral solute, with a water solvent.

In the present work - motivated by both the above mentioned solvation dynamics initial regime's importance and its varied and sometimes contradictory literature interpretations - we revisit this initial regime's mechanistic interpretation, employing the work/power energy flow methodology together with computational and analytic analysis. We focus exclusively on the standard model described above for the suddenly charged solute in a water solvent (arguably the most important solvent), and within that model, we concentrate on the issues identified above concerning appropriate characterization of the initial regime. We hasten to remark that this is of course a quite special choice of solute, solvent and model thereof. In many ways a number of its properties exaggerated compared to those for larger and more complex solutes, smaller and more diffuse charge redistributions upon excitation, and larger and more complex solvent molecules. Nonetheless, we believe that the present results will be relevant not only for solvation dynamics in water and other hydrogen bonded solvents, but also for perspective in the interpretation and discussion of solvation dynamics for the more complex systems of ever increasing interest and study.

The outline of the remainder of this paper is as follows. Our methodology is briefly outlined in Sec. II. Section III is devoted to a first discussion for the initial regime for the solvation dynamics displayed in Fig. 1, and in particular, insights provided in this context by our prior results $^{32-35}$ on the associated energy flow to water librations and translations. With this background as motivation, the dynamics of the water molecule's orientational response to the solute's suddenly acquired charge is examined, first with several indicative individual trajectory results, and subsequently with a fully averaged study of this response with the appropriate initial Maxwellian momentum distribution and - as a direct, contrasting probe of the inertial dynamics issue - with vanishing initial momentum. Attention is then turned in Sec. IV to the impact of the corresponding initial momentum comparison for the dynamical behavior of the FSF function $S(t)$. 
Section $\mathrm{V}$ then marshals analytic investigation to comprehend these results. Both the clear dominance of noninertial charge change-induced torque effects for the dynamics and the apparently contradictory success of force and torque-free inertial dynamical descriptions conflicting with that dominance are addressed, with an important conclusion on the importance of detailed energy flow examination for characterizing the molecular character of the solvations dynamics. Concluding remarks are offered in Sec. VI.

\section{METHODS}

The computations in the present work are similar to those in our previous contributions of water solvation dynamics $^{32-35}$ to which we refer for a more detailed account. The results to be presented correspond to the well known SPC/E rigid water model. ${ }^{98}$ It is to be noted that highly similar results (not reported here) have also been obtained for the flexible model used in related studies by Tran and Schwartz ${ }^{80}$ (namely the SPC/F model due to Toukan and Rahman, ${ }^{99}$ where intramolecular flexibility is achieved by adding a phenomenological intramolecular force field to the SPC model).

As for the solute, we have again adopted the same choices as Tran and Schwartz ${ }^{80}$ for ease of comparison. For the solute electronic ground state, the water-solute interaction was chosen to be identical to the water-water LJ interaction (for this water model, there are no LJ terms associated with the hydrogens, so that the solute is similar in size and mass to an oxygen atom). The electronic excited state, characterized by a positive unit charge, is simply constructed by grafting the corresponding Coulomb interaction on top of the LJ contribution.

All simulations have been run with an in-house code using one solute and 199 water molecules, which for a cutoff distance of half the box length corresponds to an interaction length of $9 \AA$. The Ewald sum correction has been included for Coulomb forces. After equilibration, calculations consists of a long trajectory (with temperature control $^{100}$ ) from which initial configurations are sampled, and then used for independent nonequilibrium runs (with no temperature control). Most of the results correspond to sets of 5,000 trajectories (with 1 fs timestep), although some correspond to sets of 10,000 trajectories (with 2 fs timestep). Finally, the first solute's solvation shell is defined as enclosing all water molecules up to a waterion separation of $3.9 \AA$. Further details will be reported within when required.

\section{INITIAL REGIME DYNAMICS: FSF DYNAMICS AND WATER MOLECULE ORIENTATIONAL RESPONSE}

\section{A. FSF Solvation Dynamics}

Here we return to the FSF solvation dynamics Fig. 1 to place our efforts in perspective, and in particular to make some first remarks on aspects of the first $20 \mathrm{fs}$ initial regime indicated there.

But it is useful first to recall the results of the first energy flow perspective applied to Fig. 1. Substantial energies in the initially created (Coulomb) energy perturbation are transferred (up to $\sim 10 \mathrm{kcal} / \mathrm{mol}$ per molecule), to the closest water molecules, i.e. in the solute's first hydration shell. Water molecules' librations (hindered rotations) account for approximately $3 / 4$ of the initially transferred energy, with roughly $2 / 3$ of the total energy flowing directly into the newly formed ion's first hydration shell, with the flow in the initial period almost exclusively into water librations. These important features are largely independent of the ion's charge sign and/or absolute value. ${ }^{32}$ These results certainly point to the key role of the water librations in the dynamics, and help to guide the remainder of this subsection.

Returning to the interpretation of the initial regime in Fig. 1, we first focus on the last of the three commonly employed descriptors for this region: inertial, Gaussian, and underdamped. The oscillatory - albeit dampedcharacter of the dynamics is unambiguously evident in Fig. 1 at least beyond the initial regime. This is clearly underdamped behavior, soon to be argued to be predominantly of water librations (hindered rotations), and so clearly dependent on the excitation-induced torques exerted on the water molecules, and therefore assuredly not inertial. To what extent the librations are already active in the initial regime is now addressed.

The initial regime in Fig. 1 has a 20 fs duration until the first relative minimum is reached. (It is noteworthy that this same feature is evident in the TCF of a water molecule's rotational kinetic energy, but not that of its translational kinetic energy. ${ }^{36}$ ) Let us consider the possible relationship of this time to the water librations. This time corresponds to half a period of an oscillation characterized by a wavenumber of $\sim 800 \mathrm{~cm}^{-1}$. This simplified assessment certainly implicates the water librations, whose spectrum falls in the $\leq 900 \mathrm{~cm}^{-1}$ range. ${ }^{101-105}$ This strongly suggests that this initial time regime is predominantly governed by torques and is thus largely noninertial in character: the dynamics evidently intimately involve the underdamped water librational motion even at this early 20 fs stage, even though no oscillations are explicitly apparent in this regime, the timescale being less than a librational period. (These same general conclusions apply in the case of a negative charge creation: see SI Sec. S1). In the next subsection, we confirm these suggestions. Indeed, we demonstrate that the solute hydration shell's water molecules are non-inertially strongly 
driven into their new orientations by the newly created torques, and not by force and torque-free inertial motion.

\section{B. Water Molecule Orientation Response}

The energy flow/power nonequilibrium dynamics results in Ref. 34 determined to which extent these charge change-induced fluxes just mentioned in Sec. III A translate into orientational and translational structural changes. As could be expected for a neutral solute acquiring a net charge, the concomitant rearrangements are substantial and - what is more remarkable - we find that most of the significant reordering is completed in a remarkably short time, roughly 20 fs (see also SI Sec. S3). In this subsection, we will consider whether initial momenta play a noticeable role in the initial regime solvation dynamics, as reflected in the water molecule orientations, or whether instead torques dominate the motion.

To this end, we will employ a straightforward-but nonetheless instructive - route to address this question, first for several indicative individual trajectories in Fig. 2 and then with a fully trajectory-averaged realization in Fig. 3; the former are consistent with, and help to provide a molecular level perspective for, the latter. For a given initial equilibrium configuration of a neutral monatomic solute, three different types of short duration (tens of fs) trajectories are generated, along the lines of the energy flow analysis methodology. ${ }^{32}$ These are respectively characterized by the following initial conditions: (1) the solute instantaneously acquires a positive unit charge; (2) the solute instantaneously acquires this same unit charge, plus all particle (solute and solvent) initial momenta are set to zero; (3) no charge is added to the solute, nor are particle momenta changed. In the indicative trajectories that we present first, finite initial momenta are drawn from an equilibrium Maxwell distribution. In the fully averaged results presented later, averaging over initial Maxwellian momenta is effected, except for case (2).

In all these cases, the orientation of the solute's immediately neighboring water molecules is monitored through the angle between the water molecule dipole moment and the water-ion direction (see sketch in Fig. 2). The indicative trajectory results following this angle are now presented in Fig. 2, should this angle follow a similar path for trajectories (1) and (3), this would indicate that the new solute charge at most feebly affects the water molecule dynamics and that it is the momenta that primarily determine the short time dynamics via approximately free streaming inertial motion; in contrast, a strong similarity between (1) and (2) would signal the relative irrelevance of initial momenta, and the pre-eminence of the non-inertial initially created torques' impact, for the initial regime dynamics.

Figure 2 displays the results for these three cases via the tracking of a given water molecule that initially belongs to the solute's first hydration shell, with the se-
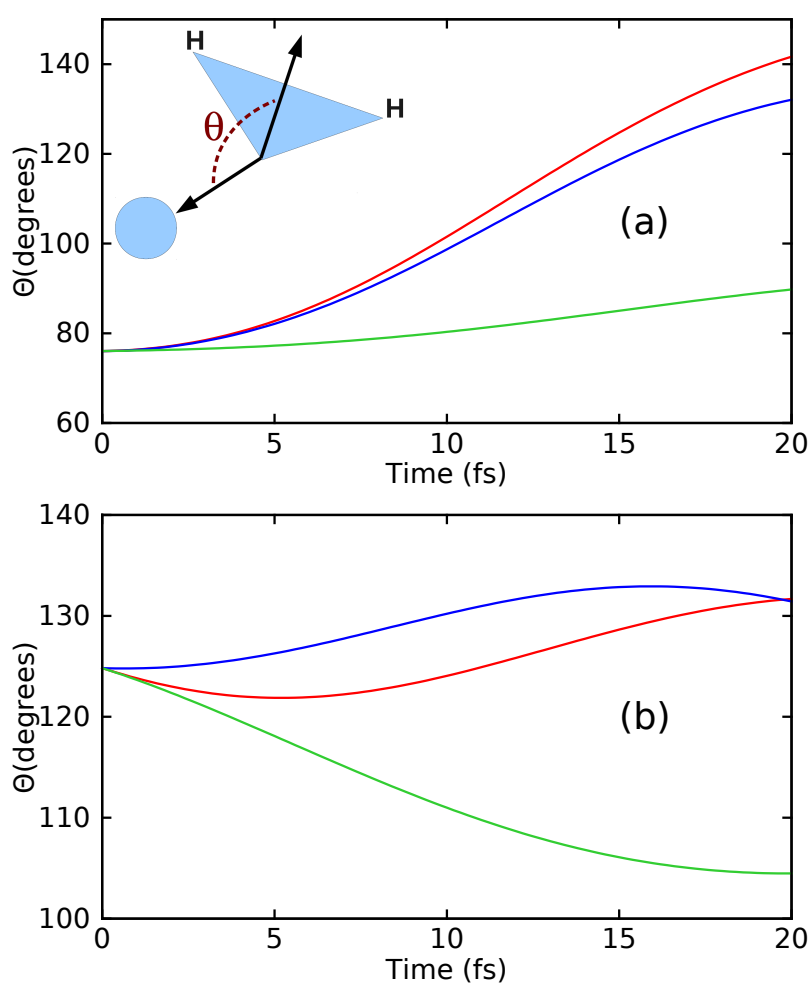

FIG. 2: Indicative trajectory results for the time dependent angle (see inset for definition related to the water molecule dipole direction) subtended by a water molecule belonging to the first hydration shell of an initially neutral monatomic solute, if: (1) blue: the solute acquires a unit charge, initial momenta are selected form a Maxwellian distribution; (2) red: idem plus all particle initial momenta are set to zero; (3) green: the solute is kept neutral and the initial momenta are again selected from a Maxwellian as in (1). The two chosen configurations in panels (a) and (b) are representative of those inspected. The equilibrium average angle for the neutral solute case is $\sim 90^{\circ}$, with a rather wide distribution, while that in the presence of the positive charge is $\sim 135^{\circ}$ for the closest hydration shell molecules, again with a wide distribution (cf SI Fig. S3(b)). The initial angle of the examined water molecule in case (a) is $\sim 75^{\circ}$, which is closer to the neutral solute equilibrium average than that for the charged solute. Conversely, the initial angle for the case (b) examined water molecule is $\sim 125^{\circ}$, which is closer to the equilibrated value of the charged solute rather than that for the neutral solute. The trajectory-averaged results will be presented in Fig. 3.

lected water molecule's initial angle in panel (a) being $\sim 75^{\circ}$ and $\sim 125^{\circ}$ in panel (b). As will be seen, all these Fig. 2 indicative trajectories display the general features exhibited by the averaged results presented in Fig. 3. Comparison of both panels' trajectories of cases (1) and (2), and of those with case (3), shows two essential features: first, that there is a marked departure for the charged solute trajectories and the neutral one, and second - specially important for the present purposesthat after 20 fs there is little difference between the orientations of the shell's water molecules, even though in 
case (2) initial momenta have been set to zero and are perforce inactive. Thus, initial momenta do not seem to play any substantial role in this initial regime: even the case (1) and (2) trajectories are similar, certainly when compared to those of the neutral solute case (3). Thus the perturbed torque field arising from the creation of the charge is evidently critical, in fact almost from the very instant of excitation, most specially in panel (a). The evident insubstantial importance of the initial momenta selected from a Maxwellian distribution is not inconsistent with the rapidly attained, large and supra-thermal, rotational kinetic energies observed for earlier sampled trajectory results on the same model system (cf Fig. 8 of Ref. 32).

Further details of Fig. 2 are instructive as well. The impact of the charge creation-induced driving torque changing the water molecule's angle is clearly larger in panel (a) - where the initial orientation is unfavorable for the created positive charge since a water hydrogen partially points towards the neutral solute - than in panel (b). In the latter situation, the initial water dipole's orientation is much closer to that appropriate in the presence of a positively charged solute.

These simple indicative trajectory results clearly point to the dominance of the charge creation-induced noninertial torque effects in the initial regime, with only minor initial momentum-dependent inertial effects possibly occurring in the very earliest portions of that regime.

Having considered for molecular perspective the limited number of trajectories just inspected in Fig. 2, we now present more extensive results averaged over $10^{4}$ trajectories, and displayed in Fig. 3. In a fashion similar to that reported in Ref. 34, the time-dependent value of the angle $(\theta(r ; t))$ is computed as a function of the distance $(r)$ from the solute ion's center, and averaged over trajectories; the resulting curves are shown for $0 \mathrm{fs}$, $10 \mathrm{fs}$, and $20 \mathrm{fs}$ after excitation, encompassing the initial regime during which a substantial portion of the orientational equilibrium is attained (see Fig. 3 caption). We compare the results for the sets of trajectories in cases (1) and (2), for which we recall that a unit charge is created, with the only difference being that all initial momentawhich now have a Maxwell distribution in case (1) - are set to zero in case (2). As is now anticipated on the basis of the results of Fig. 2, and in contrast to an inertial picture, there is hardly any difference in the two results at any time. Even though we are dealing with extremely short times, no significant effect of initial momenta is readily discernible: the impact of the newly created torque/force field supersedes any influence of initial momenta. There is a further clear indication of charge creation-induced Coulomb field-driven character of the response in Fig. 3 (further elaborated in SI Sec. S3): the magnitude of the water orientational changes far exceed those in the equilibrium fluctuation range.

Although we consider these results already compelling, we add a further relevant energy flow result for this model system which strengthens the case for the dominance of

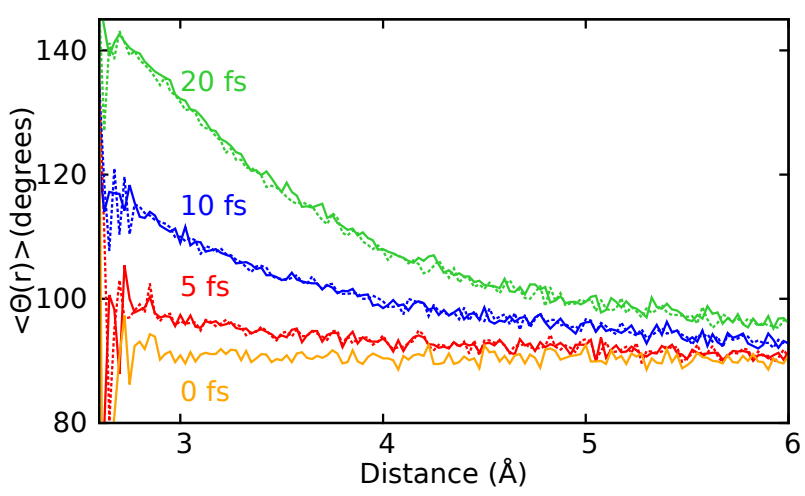

FIG. 3: Average of the time-dependent angle $\langle\theta(r ; t)\rangle$ (see inset in Fig. 2 for definition of $\theta$ ) for the water molecules as a function of distance from the solute ion of radius similar to the water molecule, at different times after excitation. The continuous curves correspond to set (1) of trajectorieswith finite initial momentum (now fully averaged) - while the dashed curves correspond to set (2), in which all initial momenta are set to zero. SI Sec. S3 shows that a key substantial portion of the final equilibrium distribution is reached in the initial time regime; further orientational rearrangements of the water molecules are the result of subsequent translational motions. It also more clearly indicates the large magnitude of the orientation changes, far outside the equilibrium fluctuation range. This is completely consistent with the rapidly attained, large and supra-thermal, rotational kinetic energies observed for the earlier sampled trajectory results in Fig. 8 of Ref. 32. (Noise is dominant at the shortest separations since these are hardly visited for the limited number of trajectories run, specially for the neutral solute.)

initial torques/forces in the initial regime. It was shown in the energy flow analysis in Ref. 34 that there is a substantial correlation between the energy channeled through each water molecule principal axis and the respective torques at the time of excitation, pointing to the initial torques as determinant for the initial regime dynamics.

\section{FSF SOLVATION DYNAMICS SIMULATIONS}

The angle $\theta$ dynamics discussed in the previous Section provides an intuitive geometrical perspective on the solvation dynamics relaxation process. But it is obviously important to check, for the key FSF function $S(t)$ (Eq. 1), whether the solvation energy dynamics is also largely insensitive to the initial momenta during the initial regime, and dominated there by torques created by the solute's sudden charge change.

For simulation study of our model of a neutral monoatomic solute that acquires a finite charge, Eq. 1 for the FSF can be more conveniently expressedby focusing on the energy gap $\Delta E$ between the excited and ground electronic states, which in our case is the 
Coulomb potential energy $V_{c}(t)$ of interaction between the solute and the water solvent molecules which appears in the excited state - as the normalized and shifted version (Eq. 2) of the ion-water Coulomb energy variation $\left(\delta V_{c}(t) \equiv V_{c}(t)-V_{c}(0)\right)$,

$$
S(t)=\frac{\overline{\delta V_{c}(t)}}{\overline{V_{c}(0)}-\overline{V_{c}(\infty)}}+1,
$$

where the overbars indicate an average over nonequilibrium trajectories. In order to describe the model experiment, the system's initial distribution will be the equilibrium distribution just prior to the excitation, i.e. for the ground electronic state with the water solvent in equilibrium with the neutral solute and all particle momenta governed by Maxwell distributions. The dynamics in the trajectories and $S(t)$ are of course those appropriate to the excited state, i.e. the water solvent molecules are subject to the electric field of the newly created solute charge.

Equation 2 indicates that we need to focus on the evolving Coulombic interaction potential energy $V_{c}(t)$, and compute its average in the excited state with the appropriate initial ground state equilibrium spatial distribution: the water solvent in equilibrium with the neutral solute. But we will probe the inertial or noninertial character of the dynamics by comparing two sets of such trajectory results with different initial momentum distributions: the first, normal one with the initial momenta properly corresponding to Maxwell distributions (with average initial temperature of $300 \mathrm{~K}$ ), and second, the reference case where those initial momenta are simply set to zero (so that inertial free streaming change of positions causing $\Delta E$ changes would not occur).

These two averages are presented in Fig. 4, corresponding to 5,000 trajectories, with a time step of $1 \mathrm{fs}$, with each trajectory 2.5 ps long (only a 0.5 ps time span is presented). Figure 4(a) shows for the FSF solvation dynamics, as was the case for the water orientational dynamics in Sec. III B, that there is hardly any short time effect stemming from the initial momenta. This is particularly the case for our focus, the initial regime ( $\sim 20 \mathrm{fs}$ ). Again the charge change-induced torques and forces are evidently dominant in this initial regime, which accordingly must be predominantly non-inertial in character. This conclusion is further emphasized in panel (b) of Fig. 4, which provides a close up view of the initial regime results. These results are completely consistent with our initial considerations in Sec. III B. (We have also included in panels (a) and (b) of Fig. 4 a quadratic time expansion approximation to $S(t)$ to be discussed presently.)

Figure 4(a) also shows that there is an effect of initial momenta in the comparison, but, remarkably, this is manifest clearly only at longer times. This effect is readily explained by the rather drastic initial perturbationkinetic, rather than potential: our comparison choice corresponds to a vanishing initial kinetic temperature. This results in an average final temperature of roughly $220 \mathrm{~K}$
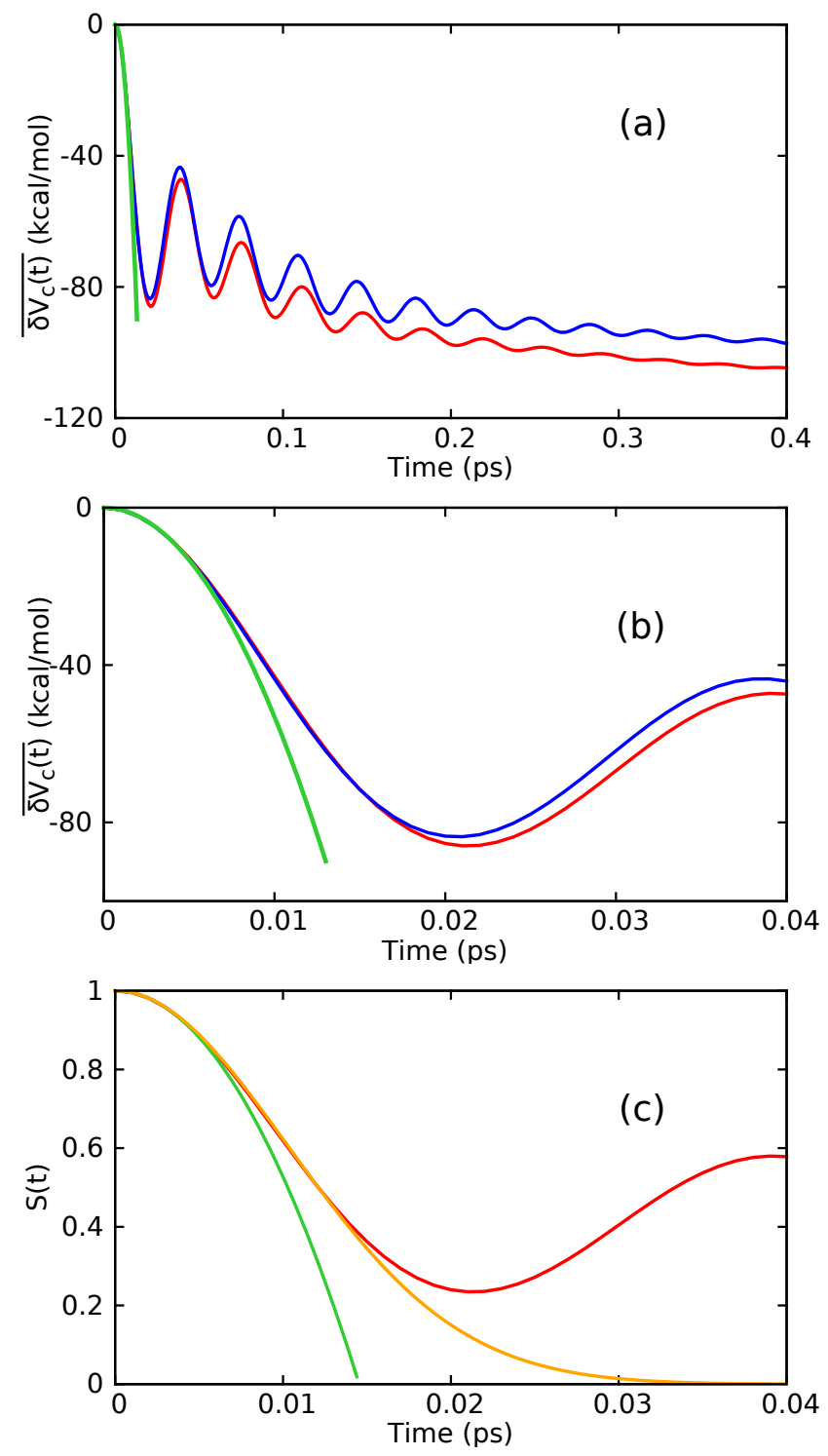

FIG. 4: (a) Trajectory results for the average time dependent Coulomb potential which enters the FSF $S(t)$ (Eq. 2), with two different conditions for the initial momenta. Red curve: $\delta V_{c}(t)$ function (see Eq. 2) for a neutral solute instantaneously acquiring a positive unit charge with a $300 \mathrm{~K}$ Maxwellian distribution for initial momenta; Blue: idem, but with all initial momenta set to zero; Green: the quadratic short time expansion developed in Sec. V. Panel (b) provides a shorter time interval blow-up of panel (a) which more clearly reveals key aspects of the initial regime dynamics discussed in the Text. (c) The FDF solvation dynamics function $S(t)$ compared to the Gaussian approximation to it (orange).

(after $2.5 \mathrm{ps}$ ); this is far below a value of $\sim 315 \mathrm{~K}$ when initial momenta are not altered, i.e. starting from an average initial $300 \mathrm{~K}$ temperature. The vanished initial momenta impede attainment of a more optimal final configuration, reflected in the final energies offset evident in Fig. 4.

Finally, panels (a) and (b) of Fig. 4 provide the initial 
quadratic behavior in time, information which is relevant to a Gaussian approximation to $S(t)$, shown in Panel (c) for comparison. These aspects will be discussed in Sec.V, after we first develop a theoretical framework for the discussion.

\section{THEORETICAL ANALYSIS}

Here we have examined, via numerical and analytic work, the solvation dynamics for a standard model system of water. We have shown that initial molecular momenta play little significant role in the initial fast solvation relaxation that follows electronic excitation of, and charge creation for, a solute. Instead, the nonequilibrium dynamics are predominantly described by "steering", i.e. motion governed by the torques generated by the newly created charge distribution.

The simulation results presented in the preceding sections for the water solvation dynamics for the model system are remarkably unambiguous on the issue of the relative muted importance of inertial aspects - governed by finite initial momenta - and the contrasting dominant importance of the non-inertial aspects-governed by the torques and forces generated by the solute's sudden charge acquisition.

In particular in the initial time regime (and sometimes beyond) these results all indicate that regime is hardly, if at all, governed by free streaming, inertial dynamics for the water molecules. Instead, each of these results - for the FSF function $S(t)$ 's dynamical behavior, including its initial quadratic behavior in time (Sec. IV), and the water reorientation dynamics (Sec. III) - paints the picture that the initial regime is predominantly, if not in fact entirely, driven by the induced torques (and forces) exerted on the water molecules surrounding the suddenly charged solute.

Let us put these results in some historical perspective. It was argued early ${ }^{12-15}$ that inertial free streaminginduced change of the energy gap $\Delta E$ could account for the initial solvation dynamics in some solvents, but this would - in hydrogen-bonded solvents such as water- be very quickly overcome, due to the rapid librational motions governed by the torques on the molecules. ${ }^{13,16,17}$ Yet, this was not an argument that there would be no inertial motion impact at all. This, plus the quite clear success of an inertial description for certain solvents, ${ }^{13,15-17}$ calls for a re-examination of the arguments in favor of any inertial motion initial contribution to the solvation dynamics, taken up next.

\section{A. Preliminaries}

In order to simplify the notation, we will employ massweighted coordinates, such that for each particle, the Cartesian coordinate is scaled by the square root of the mass and the momentum component is scaled by the inverse of that square root. The particle masses will be made explicit at the conclusion when their explicit presence is important.

It will also be useful to introduce some further notation at the beginning. The Hamiltonians of the ground $(g)$ and excited $(e)$ states are $H_{g, e}=K+V_{g, e}$, involving the system kinetic energy $K$ and total potential energy, with the excited state potential energy $V_{e}$ differing from its ground state counterpart by the Coulomb potential, $V_{e}=V_{g}+V_{c}$. Accordingly, the corresponding vector forces $\boldsymbol{F}_{g, e}=-\boldsymbol{\nabla} V_{g, e}$ differ by the Coulomb force $\boldsymbol{F}_{c}=-\boldsymbol{\nabla} V_{c}$. Finally, the Liouville operators $i L_{g, e}$ governing the ground and excited state dynamics, involving the particle momenta $\boldsymbol{p}$ and those forces are, with $i L_{g, e}$ being the Poisson bracket $\left\{A, H_{g, e}\right\}$,

$$
\begin{aligned}
i L_{e} & =\boldsymbol{p} \cdot \boldsymbol{\nabla}_{r}+\boldsymbol{F}_{e} \cdot \boldsymbol{\nabla}_{p}= \\
& =i L_{g}+\boldsymbol{F}_{c} \cdot \boldsymbol{\nabla}_{p}
\end{aligned}
$$

where we have invoked the force relation $\boldsymbol{F}_{e}=\boldsymbol{F}_{g}+\boldsymbol{F}_{c}$, and used shorthand notation for the multi-dimensional solute plus water solvent system and the convention that $\boldsymbol{A} \cdot \boldsymbol{B}$ is $\sum_{i} A_{i} B_{i}$, the sum over particles and Cartesian coordinates. The first component $\boldsymbol{p} \cdot \boldsymbol{\nabla}_{\boldsymbol{r}}$ of $i L_{e}$ (and $\left.i L_{g}\right)$ represents free streaming inertial motion independent of the forces, while the second component $\boldsymbol{F}_{e} \cdot \boldsymbol{\nabla}_{\boldsymbol{p}}$ carries the impact of the non-inertial excited state forces on the motion. It is key for our subsequent discussion that its key Coulomb contribution $\boldsymbol{F}_{c} \cdot \boldsymbol{\nabla}_{\boldsymbol{p}}$ represents the non-inertial excited state Coulomb force impact on the motion. A simple discussion explicitly illustrating this character of the terms in the Liouville operator is given in SI Sec. S6.

\section{B. Ground State TCF Interlude}

It will be useful for perspective to first consider key aspects of the original discussions which let to an inertial perspective. Early solvation dynamics discussions focused on equilibrium TCF approaches, and the principal such approaches involved two approximations. The first is that $S(t)$ can be approximated by the normalized equilibrium TCF $C_{g}(t)$ of the energy gap $\Delta E(t)$ in the ground electronic state, with both the initial distribution and the dynamics being those of that state

$$
S(t) \sim C_{g}(t)=\frac{\langle\delta \Delta E(0) \delta \Delta E(t)\rangle_{g}}{\left\langle(\delta \Delta E)^{2}\right\rangle_{g}},
$$

with the definition $\delta \Delta E(t)=\Delta E(t)-\langle\Delta E\rangle_{g}$. It is important to stress for later reference that, accordingly, the Coulomb force figures neither in the initial distribution nor the dynamics. ${ }^{106}$ The second approximation is that 
the equilibrium TCF's short time dynamics can be approximated by a Gaussian in time ${ }^{12-15,90,91,96,97}$ characterized by a ground state frequency $\omega_{g} \cdot{ }^{13,17,29}$

$$
\begin{aligned}
C_{g ; G}(t)= & e^{-\frac{\omega_{g}^{2}}{2} t^{2}} ; \\
\omega_{g}^{2}= & \frac{\left\langle(\Delta \dot{E})^{2}\right\rangle_{g}}{\left\langle(\delta \Delta E)^{2}\right\rangle_{g}}=\frac{\left\langle\left(\boldsymbol{p} \cdot \boldsymbol{\nabla}_{\boldsymbol{r}} \Delta E\right)^{2}\right\rangle_{g}}{\left\langle(\delta \Delta E)^{2}\right\rangle_{g}}= \\
= & \frac{\left\langle\boldsymbol{F}_{\boldsymbol{c}} \cdot \boldsymbol{F}_{\boldsymbol{c}}\right\rangle_{g}}{\beta\left\langle\left(\delta V_{c}\right)^{2}\right\rangle_{g}}
\end{aligned}
$$

where the second line involves the inertial Liouville operator $\boldsymbol{p} \cdot \boldsymbol{\nabla}_{\boldsymbol{r}}$, while the last line is the evaluation for the present Coulomb potential energy case, and $\beta$ is $\left(k_{B} T\right)^{-1}$.

This form is the exponentiation of the expansion of $C_{g}(t)$ through second order in time, with the quadratic term involving $\langle\delta \Delta E(0) \cdot \delta \Delta \ddot{E}(0)\rangle_{g}$. By invoking the feature that any equilibrium TCF is even in time, or equivalently by effecting an integration by parts (see SI Sec. S5, Eq. S9 et seq.), this quadratic term average becomes $\left\langle(\delta \Delta \dot{E})^{2}\right\rangle$, the form appearing in Eq. 5 (specification of the common initial time $t=0$ is unnecessary in the equilibrium average. ${ }^{107}$ ) Again, we stress that the initial time derivative evaluated here is $i L_{g} \delta \Delta E=\boldsymbol{p} \cdot \nabla_{\boldsymbol{r}} \Delta E$, i.e. the initial inertial change for $\Delta E$ (we continue to use mass weighted coordinates). The energy gap, i.e. the Coulomb potential, is changed due to the particle coordinate changes, themselves due to the free streaming motion of the particles, un-influenced by any force.

The important result for present purposes is that the equilibrium $C_{g ; G}(t)(t)$ was shown to be - surprisinglya very good approximation for a significant fraction $(\sim$ $2 / 3$ ) of the nonequilibrium $S(t)$ 's decay for the modeled non-hydrogen bonding polar solvent $\mathrm{CH}_{3} \mathrm{Cl}$. Similar success of a Gaussian time dependence for $S(t)$ was found by Maroncelli for acetonitrile $\left(\mathrm{CH}_{3} \mathrm{CN}\right)$ solvent, ${ }^{14,15,30}$ and by other authors for other solvents. ${ }^{90,91,96,97}$ This success rather strongly supported the inertial perspective: the dominant effect is of the solvent molecules' initial free streaming motion, changing their positions and thus the energy gap. However - as anticipated in Ref. 13 and simultaneously found in a methanol $\left(\mathrm{CH}_{3} \mathrm{OH}\right)$ solvent simulation by Fonseca and Ladanyi ${ }^{16,17}$ — this dominance would be negated by the early and marked departure for aqueous and other strongly hydrogen-bonded solvents with rapid librational motions, i.e. motion driven by transition-induced torques and forces.

These observations were mainly directed to equilibrium TCF approximations to the dynamics. A detailed analysis for the nonequilibrium dynamics is considered next.

\section{Short Time Regime Focus}

With these preliminaries, we start with the required nonequilibrium average of the Coulomb potential at time $\mathrm{t}$, which is

$$
\begin{aligned}
\overline{V_{c}(t)} & =\int d \Gamma\left[e^{-i L_{e} t} \rho_{g}\right] V_{c} \\
& =\int d \Gamma \rho_{g} e^{i L_{e} t} V_{c}=\left\langle e^{i L_{e} t} V_{c}\right\rangle_{g},
\end{aligned}
$$

where the brackets indicate the phase space average with the initial ground state equilibrium distribution $\rho_{g} \propto e^{-\beta H_{g}}$.

A straightforward expansion in powers of time of Eq. 6 has no term linear in time, since the first order change $i L_{e} V_{c}=\boldsymbol{p} \cdot \boldsymbol{\nabla} V_{c}$ is odd in momentum and the average vanishes. The leading order time-dependent contribution to $\overline{\delta V_{c}(t)}=\overline{V_{c}(t)-V_{c}(0)}$ is then the quadratic expression

$$
\begin{aligned}
\overline{\delta V_{c}(t)} & =\frac{t^{2}}{2}\left\langle\left(i L_{e}\right)^{2} V_{c}\right\rangle_{g}=-\frac{t^{2}}{2}\left\langle\left(i L_{e}\right) \boldsymbol{p} \cdot \boldsymbol{F}_{c}\right\rangle_{g}= \\
& =\left(-\frac{t^{2}}{2}\right)\left[\left\langle\left(i L_{g}\right) \boldsymbol{p} \cdot \boldsymbol{F}_{c}\right\rangle_{g}+\left\langle\left(\boldsymbol{F}_{c} \cdot \boldsymbol{\nabla}_{\boldsymbol{p}}\right) \boldsymbol{p} \cdot \boldsymbol{F}_{c}\right\rangle_{g}\right]= \\
& =-\frac{t^{2}}{2}\left\langle\boldsymbol{F}_{\boldsymbol{c}} \cdot \boldsymbol{F}_{\boldsymbol{c}}\right\rangle_{g} .
\end{aligned}
$$

The second line follows from the decomposition of $i L_{e}$, eq. 3 , into its ground and excited state contributions. The first contribution vanishes by integration by parts to produce $i L_{g} \rho_{g}$, which vanishes since the ground state dynamics do not alter the equilibrium distribution $\rho_{g}$; this and the evaluation of the second term gives the final line, involving the square of the Coulomb force. (Note that one factor of the Coulomb potential-through the force $\boldsymbol{F}_{c}$-must enter simply because the non-equilibrium average is of that potential, while the other factor requires the impact of dynamics) . The derivation just presented is repeated in somewhat more detail in SI Sec. S4. Incidentally, the average in Eq. 7 without mass-weighted coordinates is $\left\langle\sum_{i}\left|\boldsymbol{F}_{c_{i}}\right|^{2} / m_{i}\right\rangle_{g}$, where the $i$ index runs over all particles, implying that an isotopic effect is to be expected.

We stress at this stage that the average in the final form in Eq. 7 has a non-inertial character, which can be understood as follows. This average results from the excited state Liouville operator Coulomb force component $\boldsymbol{F}_{c} \cdot \nabla_{p}$ acting on $\boldsymbol{p} \cdot \boldsymbol{F}_{c}$. We already emphasized that this component represents non-inertial motion related to the suddenly created solute charge. We can make this connection even more explicit: we give a simple but general demonstration in SI Sec. S6 that this component of the Liouville operator describes the non-inertial forceinduced change in initial momentum - the accelerationof a particle, in our case, of the surrounding solvent water molecules responding to the switched-on Coulomb force $\boldsymbol{F}_{c}$. 
It will prove important for later reference to observe that Eq. 7-as shown in SI Sec. S4 - can be written in a different form:

$$
\begin{aligned}
\overline{\delta V_{c}(t)} & =-\frac{t^{2}}{2} \beta\left\langle\left(\boldsymbol{p} \cdot \nabla_{\boldsymbol{r}} V_{c}\right)^{2}\right\rangle_{g}= \\
& =-\frac{t^{2}}{2} \beta\left\langle\boldsymbol{p} \boldsymbol{p}: \boldsymbol{F}_{c} \boldsymbol{F}_{c}\right\rangle_{g} .
\end{aligned}
$$

This is readily obtained form the first line of Eq.7 by integrating by parts the complete $i L_{e}$ operator. This generates $i L_{e} \rho_{g}$, whose $i L_{g} \rho_{g}$ contribution vanishes (as above), leaving the term $-\beta \rho_{g} \boldsymbol{p} \cdot \nabla_{\boldsymbol{r}} V_{c}$. (The steps are explicitly followed in SI Sec. S4). The new form Eq. 8 of the quadratic term is clearly of an inertial character, involving as it does only the inertial free streaming operator $\boldsymbol{p} \cdot \boldsymbol{\nabla}_{\boldsymbol{r}}$.

Equations 7 and 8 apparently provide conflicting descriptions of the initial dynamics: the non-inertial character of Eq. 7 -in accord with the earlier presented strong evidence of the dominance of non-inertial charge change-induced Coulomb forces in the motion - evidently at odds with the inertial character of Eq. 8. We will see that these two equations prove to be instructive in interpreting our simulation results and the resolution of the apparent conflict. We stress here that the results in these two equations as well as in Eq. 5, all involve the same inverse mass dependence when returned to regular coordinate and momenta definitions. (In all the equations above, we have left momentum terms unaveraged in order to facilitate that discussion. The observant reader might note that, while apparently differing in an unaveraged form, Eqs. 7 and 8 are identical when that average is performed; more on that later).

Before proceeding, we note that the properly normalized form of the solvation dynamics FSF $S(t)$, Eq. 2, also involves the denominator factor $\overline{V_{c}(0)-V_{c}(\infty)}$. The first term is just the ground state equilibrium average $\left\langle V_{c}\right\rangle_{g}$, while the subtracted term will be the excited state equilibrium average $\left\langle V_{c}\right\rangle_{e}$. In precisely the same maneuver used in obtaining the TCF Eq. 4 denominator, ${ }^{13,45}$ the excited state equilibrium distribution $\rho_{e}$ is approximated by $\rho_{g} \cdot\left(1-\beta \delta V_{c}\right)$, this gives

$$
\overline{V_{c}(0)-V_{c}(\infty)}=\beta\left\langle\left(\delta V_{c}\right)^{2}\right\rangle_{g} .
$$

This (minor) approximation is common to $S(t)$ and all TCF expressions in this work, so it has no impact on any difference between them.

\section{Initial Regime Analysis}

With this background, we now return to the Fig. 4 results for the present problem, where $\Delta E(t)=V_{c}$, the Coulomb potential energy. We will primarily be concerned with panels (b) and (c) of that Figure. The spe- cific features to be discussed are first, the early departure of $S(t)$ in Figure 4(c) from its Gaussian approximation, and second, the short time agreement of the quadratic approximation (which provides the information for the Gaussian approximation) in panel (b), for both the Maxwellian distribution and the vanishing of the initial momentum. Altogether, these features will demonstrate the quadratic approximation and thus the related Gaussian is - despite prior invocations - unable to distinguish between inertial and non-inertial pictures, and thus that further analysis - such as has been provided by the work/power perspective used in this workis necessary to make that distinction.

We first observe that Fig. 4(c) shows that $S(t)$ is well described by a Gaussian approximation for half of the initial 20 fs regime. From an inertial perspective, this is actually surprising, since strong interactions would be expected to induce a rapid departure from free streaming motion for aqueous and H-bonded solvents, ${ }^{13,16,17}$ as described at the beginning of this Section. But the power/work orientational calculations of Sec. III B show that strong Coulombic torques driving non-inertial motion are in fact operative in this time regime, confirming the suggestions given in Sec. III A. Indeed, on a short time scale, librational motion exhibits Gaussian behaviour. $^{25}$

However, closer inspection will reveal an unexpected and even more general and substantial difficulty: initial Gaussian behavior by itself cannot in fact be used to distinguish between inertial and non-inertial dynamics.

To see this, we need to go through a certain amount of analysis. To begin, we write out the Gaussian approximation $S_{G}(t)$ for the nonequilibrium $S(t)$ straightforwardly developed in detail in SI Sec. S5:

$$
\begin{aligned}
S_{G}(t) & =e^{-\frac{\omega_{e}^{2}}{2} t^{2}} ; \\
\omega_{e}^{2} & =\frac{\left|\left\langle\ddot{V}_{c}\right\rangle_{g}\right|}{\beta\left\langle\delta V_{c}^{2}\right\rangle_{g}}=\frac{\left\langle\left(\dot{V}_{c}\right)^{2}\right\rangle_{g}}{\left\langle\delta V_{c}^{2}\right\rangle_{g}}
\end{aligned}
$$

Here we have written two equivalent forms of the excited state square frequency $\omega_{e}^{2}$ in the second line, derived explicitly in SI Sec. S5. The first follows straightforwardly from the direct expansion of $\left\langle\left(i L_{e}\right)^{2} V_{c}\right\rangle_{g}$; this explicitly involves the coefficient of $t^{2} / 2$ in Eq. 7 and thus has a non-inertial character. The second form explicitly involves the coefficient of $t^{2} / 2$ in Eq. 8, which is instead inertial in character and was itself simply obtained by integration by parts of the first form.

At this point, we observe the difficulty; the recurrence of the apparent conflict between the quadratic terms Eqs. 7 and 8, now transferred to the Gaussian approximations to the non-equilibrium FSF $S(t)$, but now complicated by comparison with the equilibrium ground state TCF Gaussian—and supposedly inertial-result Eq. 5. We go through this contradictory situation step-wise. The second form of Eq. 10, in accord with the inertial charac- 
ter of Eq. 8, gives exactly the same result for the nonequilibrium case as does the classic equilibrium ground state TCF result Eq. 5, which indicates inertial behavior: $\omega_{e}^{2}=\omega_{g}^{2}$ ! This conclusion seems to confirm an inertial picture for $S(t)$ 's short time behavior, but - to repeat - it is in opposition to the extensive support of a non-inertial dynamics interpretation just reviewed at this subsection's beginning. In particular, it evidently contradicts all three panels of Fig. 4, most clearly in panels (b) and (c); these indicate the short time equality of $S(t)$ calculated with an initial Maxwell distribution for the momenta or zero initial momenta, thus ruling out an inertial perspective, and indicating the non-inertial dominance of the charge change-induced torques.

This seeming conundrum can be resolved by the fact that the two forms Eqs. 7 and 8-which paint opposite pictures for the character of the dynamics, non-inertial and inertial respectively - are in fact equivalent. Thus they cannot be used to unambiguously identify the dynamics' character, either for the nonequilibrium solvation dynamics FSF $S(t)$ or the equilibrium TCF. We now show in more detail that this is the case.

We begin by recalling the equivalent form Eq. 7 (i.e. equivalent to Eq. 8) employed for the calculation of $\omega_{e}^{2}$, which involves - for both the initial Maxwellian momentum distribution and vanishing momentum - the average $\left\langle\boldsymbol{F}_{\boldsymbol{c}} \cdot \boldsymbol{F}_{\boldsymbol{c}}\right\rangle_{g}$, clearly highlighting — via the presence of the initial excited state Coulomb force and the absence of the momentum - the non-inertial role of the charge change-induced initial forces and torques. Indeed it was this average $\left\langle\boldsymbol{F}_{\boldsymbol{c}} \cdot \boldsymbol{F}_{\boldsymbol{c}}\right\rangle_{g}$ form (rather than Eq. 8) that we used to give the initial quadratic $t^{2}$ behavior displayed in panels (b) and (c) of Fig. 4, and this is clearly in numerical agreement with the initial quadratic behavior of $S(t)$ with vanishing initial momenta: the same noninertial quadratic time dependence applies for zero initial momenta and a Maxwellian distribution. ${ }^{108}$ On the other hand, if one only focused on the argument of Eq. 8 , that would vanish for initial momenta; in this connection, here we recall the indicative trajectory results in Fig. 2 where no momentum average was effected, and a non-inertial perspective was supported. But of course, as we explicitly noted in our first discussion of Eqs. 7 and 8 above, if the momentum average is carried out in Eq. 8, one obtains exactly Eq. 7.

We can briefly summarize the relevant aspects of the nonequilibrium Coulomb potential case quadratic time and allied Gaussian approximation behavior as follows.

The non-inertial short time dominance for torques and forces are indicated by the results that: (i) Eq. 7 gives the same, correct numerical, result for the zero momentum and Maxwell-averaged initial quadratic $t^{2}$ behavior of $S(t)$ in Figs. 4(b) and 4(c); (ii) at the same time, the non-inertial perspective Eq. 7 leads to the square frequency $\omega_{e}^{2}$ - the first form in Eq. 10 - for the appropriate nonequilibrium Gaussian approximation $S_{G}(t)$ to $S(t)$ in Fig. 4(c).

But at the same time, the contrasting inertial perspec- tive for the dynamics are indicated by the results that: (iii) the square frequency $\omega_{e}^{2}$-the second form in Eq. 10 - calculated from the inertial form Eq. 8 gives the same numerically correct results for the zero momentum and Maxwell-averaged initial quadratic $t^{2}$ behavior of the nonequilibrium $S(t)$ in Figs. 4(b) and 4(c)-compare item (i) above; and (iv) this inertial form also gives the identical appropriate Gaussian approximation $S_{G}(t)$ Eq. 10 to the nonequilibrium $S(t)$ in Fig. 4(c) - compare item (ii); and finally (v) from SI Sec. S5 and the discussion below Eq. 10, the square frequencies in the excited and ground electronic states are identical, $\omega_{e}^{2}=\omega_{g}^{2}$; thus the Gaussian $S_{G}(t)$ is (remarkably) identical to the inertial perspective ground state equilibrium TCF Gaussian result $S_{G, g}(t)$, Eq. 5 , involving the frequency $\omega_{g}$, use of the latter for the former is thus not an approximation. ${ }^{109}$

The thorough-going equivalence for the nonequilibrium Coulomb potential case just demonstrated in detail leads to a simple if unexpected conclusion: the predictions of the short time quadratic $t^{2}$ behavior of $S(t)$ and the short time Gaussian approximation $S_{G}(t)$ for $S(t)$ are identical from both the inertial and non-inertial perspectives, and cannot be distinguished, a feature already explicitly evident if one simply carried out the momentum average in Eq. 8, which generates exactly the Eq. 7 result. Thus, an unambiguous nonequilibrium short time microscopic physical description cannot be assigned. The same conclusion applies in the approximate equilibrium TCF perspective, early TCF Gaussian arguments (see e.g. Refs. 13-15) were thus necessary but not sufficient for that purpose. $^{110}$

This raises the obvious question: can the subsequent evolution of the nonequilibrium FSF $S(t)$ itself clearly distinguish these two descriptions? But the question should be posed not only in the post-Gaussian portion of the initial regime, but also in terms of their longer time successor descriptions, since clearly, even if free streaming motion were initially dominant, the motion's character must be quickly converted by the impact of any existing torques and forces. Since our present system ground and excited state interactions differ only by the Coulomb potential, the question is then: can the post-initial regime evolution of $S(t)$ distinguish between non-inertial motion driven by the charge change-created Coulomb torques and forces and either - at sufficiently early times - inertial motion, or - at later times - motion impacted by the non-Coulomb interactions common to both electronic states?

Figure 5 gives some perspective on this issue, since it considers a different case in which a smaller charge is initially created on the solute. Before discussing the details of the Figure, we consider first some issues concerning the impact of any change in the initially created Coulomb field. The standard ground state equilibrium TCF approximation Eq. 4-in which the Coulomb field presence in the dynamics are simply ignored - is obviously of no use here: the dynamics of $S(t)$ would be forced to be independent of the suddenly acquired so- 


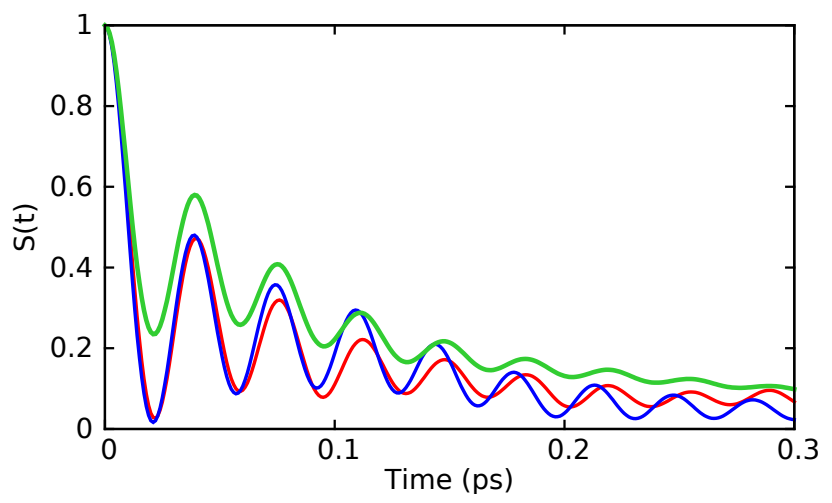

FIG. 5: Comparison of the nonequilibrium dynamics simulation results for the FSF function $S(t)$ for a neutral solute acquiring a positive charge with two disparate possible values. Red curve: instantaneous acquisition of a $0.1 e$ charge, with a $300 \mathrm{~K}$ Maxwellian distribution for initial momenta; Blue: idem, but with all initial momenta set to zero; Green: acquisition of a $1 e$ positive charge with, as for the initial case, Maxwellian initial momenta.

lute charge $\Delta q$. For the correct, i.e. unapproximated nonequilibrium $S(t)$, from Eqs. 2, 7 and 9, $S(t)$ is independent of $\Delta q$ through second order in time (the source of the lack of inertial/noninertial behavioral distinction, also reflected in the nonequilibrium Gaussian approximation): the impact of the newly created solute Coulomb potential will set in subsequently, as we now examine.

To probe the impact of the Coulomb potential, Fig. 5 compares $S(t)$ for our solute, water solvent model' s standard charge change $\Delta q=1$ (green) with a much smaller created charge $\Delta q=0.1$ (red), both with initial Maxwellian momentum distributions. The previously concealed noninertial impact of the Coulomb field is already revealed by $\sim 20 \mathrm{fs}$, as the $\Delta q=0.1$ case departure form the $\Delta q=1$ case becomes evident. In addition, the dominance of the non-inertial dynamics for the small created charge is reinforced by the comparison with the result of the reference zero initial momenta calculation (blue): the $S(t)$ results coincide up through at least half of the initial regime.

The power/work approach results for the energy flow are shown in Fig. 6, and confirm the $\Delta q=0.1$ case non-inertial driven motion, even though the amount of energy transfer is about two orders of magnitude lower than our standard case (cf. Fig. 6 caption; note that the results are normalized in order to effect the comparison). The transfer is largely to the water librations, just as for our standard charge transfer $\Delta q=1$ case; indeed the librations are even more pronounced in the smaller energy transfer case. The results and discussion of Figs. 5 and 6 provide an illustration that, while the calculation of the nonequilibrium $S(t)$ is already far more revealing than that of the ground state equilibrium TCF, comprehension of the essential character of the dynamics, over a wide range of times, will require analysis beyond the simple calculation of $S(t)$.

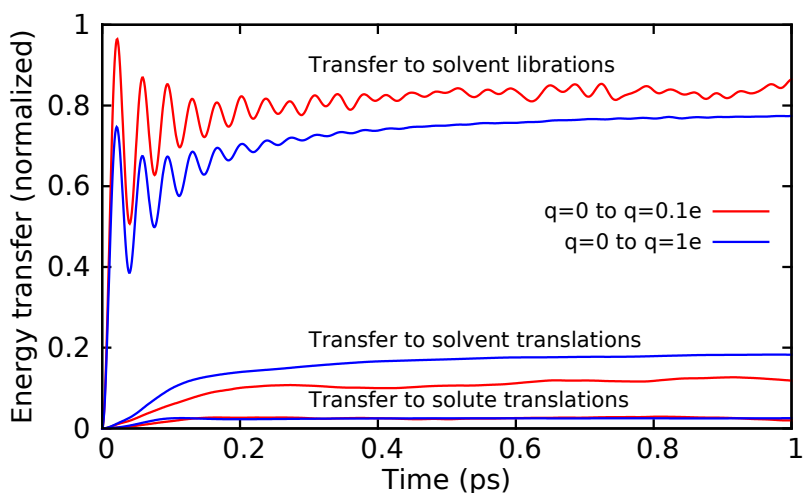

FIG. 6: Energy transfer to water solvent librations and translations and to solute translation for the charge changes 0 to $0.1 e$ (red) and 0 to $1 e$ (blue). For these two cases, the energies have been separately normalized by the total energy transfer magnitudes (kcal/mol): 0.98 (red) and 112 (blue). The total energy transfers in the initial time regime $20 \mathrm{fs}$ are in $\mathrm{kcal} / \mathrm{mol}: 0.94$ (red) and 85 (blue).

Our results in this subsection for the initial regime have a very important general implication: since the short time Gaussian behavior conceals the microscopic character of the motion, the detailed microscopic energy flow and momentum variation exercises carried out in this work were in fact necessary to conclude that it is the non-inertial excitation charge creation driven torque effects that are dominant in the initial regime of the solvation dynamics. There is also an important implication for longer times outside of the initial regime. Both our power/work based orientational results in SI Sec. S3which extend our initial regime results in Sec. IIIand the Coulomb potential impact results in Figs. 5 and 6 for initial Maxwell distributed- and vanishing momenta, have indicated that a power/work analysis - or related approaches ${ }^{111}$ — would be required to provide a more molecular level incisive description of the dynamics underlying the FSF solvent dynamical function $S(t)$.

\section{CONCLUDING REMARKS}

Here we have revisited - for the special but standard ground to excited electronic state transition model of an instantly charged solute immersed in water solvent- the mechanistic interpretation of the initial time regime of the nonequilibrium solvation dynamics induced by the charge change, of the type probed experimentally in timedependent fluorescence Stokes shift spectroscopy. As noted in the Introduction, this regime is of special interest in connection with its sometimes very significant contribution to the overall dynamics and its frequent relevance for the solvent dynamical influence on chemical reaction rates. In this effort, we have employed computational and analytic analysis, utilizing and informed by 
work/power energy flow methodology. We have shown that these dynamics are clearly non-inertial, driven by the Coulombic torques and forces on the solute's water molecule neighbors, generated by the sudden appearance of the solute charge in the excited electronic state. This contrasts with a inertial mechanism, in which the dynamics would instead be determined by the initial momenta, i.e. by the free streaming motion - unaffected by the charge change - which would alter the Coulomb potential energy gap by changing molecular positions.

We have also shown that an initial Gaussian time dependence for these nonequilibrium dynamics - while quickly departing from the actual dynamics - coincides exactly with a common approximation of a ground electronic state time correlation function (TCF), which is readily (and conventionally) interpreted as indicating inertial dynamics. Thus, any initial Gaussian time behavior - equilibrium or nonequilibrium - is unable to distinguish between, and thus to identify, the molecular mechanism - non-inertial or inertial - of the solvation dynamics. On the other hand, the work/power energy flow methodology is able to identify the mechanism in this initial time region and beyond, and to provide detailed microscopic insight on the non-inertial dynamics driven by the excitation charge created Coulomb torques and forces. And as we argued in concluding Sec. V, our results for longer times outside of the initial regime indicate that a power/work analysis - or related approaches - are requisite to generate a more molecular level characterization of the dynamics underlying the FSF solvent dynamical function $S(t)$.

Our simulations were all for the special model where the energy gap $\Delta E$ between excited and ground electronic states was the Coulomb potential energy between the charged solute and the water molecules. However, with the definition that the excited (e) and ground (g) state Hamiltonians are related by $H_{e}=H_{g}+\Delta E$, the analysis in this paper carries through unchanged. This generality is important, because as emphasized already in the Introduction, the current solute, charge creation, and water solvent model is a special one. Other solute and solvent systems will of course exhibit different detailed molecular level characteristics in the initial regime, and on succeeding time scales as well. As we point out at the conclusion of SI Sec.S4, our formulations and analysis, both for the nonequilibrium FSF and its Gaussian behavior, also hold for non-Coulombic interactions and cases where the solute size changes. Of course, the early time relative importance of non-inertial and inertial motion will likely vary from case to case. There are obviously many systems that might be considered for comparable study with the present methods. The extensive listing of the broad range of solvation dynamics arenas gives an idea of assorted possibilities, but an interesting first choice could be a funda- mental charge transfer photoreaction, ${ }^{53}$ or a system with quite strongly contrasting interactions, which might involve nonpolar solvation dynamics and solute size change where electrostriction and translational dynamics are evidently important. ${ }^{81}$ (Such effects are absent in the present system in the initial period: significant translational motion only sets in at longer time scales. ${ }^{32}$ )

All of our simulations and analysis have employed classical mechanics for the nuclei. Comparable water solvent solvation dynamics simulations for $S(t)$ instead employing quantum mechanics have found deviations below $20 \%$ from classical behavior. ${ }^{44}$ Such comparatively modest quantum effects are common for water dynamical problems, but for our present purposes, it is worth pointing out that, for such strongly interacting systems, mechanistic perspectives are typically unaltered. ${ }^{112-114}$

As noted above, a work/power energy flow analysis proves to be necessary to identify the character of the dynamics in the initial time period of the solvent relaxation. Further, it has been indicated that such an analysis can be also quite effective in this regard on other time scales; it, or something related, ${ }^{111}$ will in fact be necessary to provide a detailed microscopic description of the solvation dynamics on all time scales (other, related illustrations of this can be found in Refs. 32,36,38); novel experimental probing of the dynamics' character - especially either inertial or non-inertial, i.e. induced (forced) on short time scales - will be key in this effort as well. ${ }^{115}$ With this feature in mind, we have indicated in SI Sec. S7 several potentially useful key general power relationships that may prove useful; these connect certain time derivatives of FSF functions of the type Eq. 2-but with a general energy gap $\Delta E$ - to the average timedependent nonequilibrium power and to a quasi-time correlation function for that power.

\section{SUPPLEMENTARY INFORMATION}

The Supplementary Information associated with this article deals with the following topics: Solvation dynamics for negative charge creation; Initial decay for the created anion case; Time evolution of orientational and translational structure; Derivation of quadratic expansion terms; Gaussian time dependent solvation dynamics; Simple interpretation of Liouville operator terms; Work/power related forms.

\section{Acknowledgments}

This work was supported by MICINN grant PGC2018099277-B-C21 (RR), and NSF grant CHE-1112564 (JTH). 
* Electronic address: rosendo.rey@upc.edu

$\dagger$ Electronic address: chynes43@gmail.com

\section{REFERENCES}

1 Bagchi, B. Dynamics of Solvation and Charge-Transfer Dynamics in Liquids. Ann. Rev. Phys. Chem. 1989, 40, 115-141.

2 Maroncelli, M.; Macinnis, J.; Fleming, G.R. PolarSolvent Dynamics and Electron-Transfer Reactions. Science 1989, 243, 1674-1681.

3 Fleming, G.R.; Wolynes, P.G. Chemical Dynamics in Solution. Phys. Today 1990, 43, 36-43.

${ }^{4}$ Stratt, R.M.; Maroncelli, M. Nonreactive Dynamics in Solution: The Emerging View of Solvation Dynamics and Vibrational Relaxation. J. Phys. Chem. 1996, 100, 1298112996.

5 Fleming, G.R.; Cho, M.H. Chromophore-Solvent Dynamics. Ann. Rev. Phys. Chem. 1996, 47, 109-134.

${ }^{6}$ Cho, M.; Fleming, G.R. Electron Transfer and Solvent Dynamics in Two- and Three-State Systems. Adv. Chem. Phys. 1999, 10\%, 311-370.

7 Nandi, N.; Bhattacharyya, K.; Bagchi, B. Dielectric Relaxation and Solvation Dynamics of Water in Complex Chemical and Biological Systems. Chem. Rev. 2000, 100, 2013-2045.

8 Bagchi, B.; Jana, B. Solvation Dynamics in Dipolar Liquids. Chem. Soc. Rev. 2010, 39, 1936-1954.

9 Impey, R.W.; Madden, P.A.; McDonald, I.R. Spectroscopic and Transport Properties of Water. Mol. Phys. 1982, 46, 513-539.

10 Maroncelli, M.; Fleming, G.R. Computer Simulation of the Dynamics of Aqueous Solvation. J. Chem. Phys. 1988, 89, 5044-5068.

11 Bader, J.S.; Chandler, D. Computer Simulation of Photochemically Induced Electron Transfer. Chem. Phys. Lett. 1989, 158, 501-504.

12 Hynes, J.T.; Carter, E.A.; Ciccotti, G.; Kim, H.J.; Zichi, D.A.; Ferrario, M.; Kapral, R. In Perspectives in Photosynthesis; Jortner, J., Pullman, B., Eds.; Kluwer: Dordrecht, 1990), pp 133-148.

13 Carter, E.; Hynes, J.T. Solvation Dynamics for an Ion Pair in a Polar Solvent: Time-Dependent Fluorescence and Photochemical Charge Transfer. J. Chem. Phys. 1991, 94, 5961-5979.

14 Maroncelli, M. Computer Simulations of Solvation Dynamics in Acetonitrile. J. Chem. Phys. 1991, 94, 20842103.

15 Maroncelli, M. The Dynamics of Solvation in Polar Liquids. J. Mol. Liq. 1993, 57, 1-37.

16 Fonseca, T.; Ladanyi, B.M. Breakdown of Linear Response for Solvation Dynamics in Methanol. J. Phys. Chem. 1991, 95, 2116-2119.

17 Fonseca, T.; Ladanyi, B. Solvation dynamics in methanol: solute and perturbation dependence. J. Molec. Liq. 1994, 60, 1-24.

18 Muiño, P.L.; Callis, P.R. Hybrid Simulations of Solvation Effects on Electronic Spectra: Indoles in Water. J. Chem. Phys. 1994, 100, 4093-4109.
19 Barnett, R.B.; Landman, U.; Nitzan, A. Relaxation Dynamics Following Transition of Solvated Electrons. J. Chem. Phys. 1989, 90, 4413-4422.

20 Jimenez, R.J.; Fleming, G.R.; Kumar, P.V.; Maroncelli, M. Femtosecond Solvation Dynamics in Water. Nature 1994, 369, 471-473.

21 Perera, L.; Berkowitz, M.L. Dynamics of Ion Solvation in a Stockmayer Fluid. J. Chem. Phys. 1992, 96, 3092-3101.

${ }^{22}$ Roy, S.; Bagchi, B. Solvation Dynamics in Liquid Water. A Novel Interplay between Librational and Diffusive Modes. J. Chem. Phys. 1993, 99, 9938-9943.

${ }^{23}$ Nandi, N.; Roy, S.; Bagchi, B. Ionic and Dipolar Solvation Dynamics in Liquid Water. Proc. Indian Acad. Sci. (Chem. Sci.) 1994, 106, 1297-1306.

24 Nandi, N.; Roy, S.; Bagchi, B. Ultrafast Solvation Dynamics in Water: Isotope Effects and Comparison with Experimental Results. J. Chem. Phys. 1995, 102, 13901397.

${ }^{25}$ van der Zwan, G.; Hynes, J.T. Time Dependent- Fluorescence Solvent Shifts, Dielectric Friction, and Nonequilibrium Solvation in Polar Solvents. J. Phys. Chem. 1985, 89, 4181-4188.

26 Calef, D.F.; Wolynes, P.G. Classical solvent dynamics and electron transfer. II. Molecular aspects. J. Chem. Phys. 1983, 78, 470-482.

27 Wolynes, P.G. Linearized microscopic theories of nonequilibrium solvation. J. Chem. Phys. 1987, 86, 5133-5136.

28 Bruehl, M.; Hynes, J.T. Dielectric Friction and Solvation Dynamics: A Molecular Dynamics Study. J. Phys. Chem., 1990, 96, 4068-4074.

29 Carter, E.A.; Hynes, J.T. Solute-Dependent Solvent Force Constants For Ion Pairs and Neutral Pairs in a Polar Solvent. J. Phys. Chem., 1989, 93, 2184-2187.

30 Rosenthal, S.J.; Xie, X.; Du, M.; Fleming, G.R. Femtosecond solvation dynamics in acetonitrile: Observation of the inertial contribution to the solvent response. J. Chem. Phys. 1991, 95, 4715-4718.

${ }^{31}$ Maroncelli, M.; Kumar, V.P.; Papazyan, A. A Simple Interpretation of Polar Solvation Dynamics. J. Phys. Chem. 1993, 97, 13-17.

${ }^{32}$ Rey, R.; Hynes, J.T. Solvation dynamics in liquid water. I. Ultrafast energy fluxes. J. Phys. Chem. B, 2015, 119, 7558-7570.

${ }^{33}$ Rey, R.; Hynes, J.T. Solvation Dynamics in Water: 2. Energy Fluxes on Excited- and Ground-State Surfaces. $J$. Phys. Chem. B 2016120 11287-11297.

${ }^{34}$ Rey, R.; Hynes, J.T. Solvation Dynamics in Liquid Water. III. Energy Fluxes and Structural Changes. J. Phys. Chem. B 2017121 1377-1385.

35 Rey, R.; Hynes, J.T. Translational versus rotational energy flow in water solvation dynamics. Chem. Phys. Lett. 2017683 483-487.

36 Rey, R.; Ingrosso, F.; Elsaesser, T.; Hynes, J.T. Pathways for $\mathrm{H}_{2} \mathrm{O}$ Bend Vibrational Relaxation in Liquid Water. $J$. Phys. Chem. A 2009, 113, 8949-8962.

37 Rey, R.; Hynes, J.T. Tracking Energy Transfer from Excited to Accepting Modes: Application to Water Bend Vibrational Relaxation. Phys. Chem. Chem. Phys. 2012, 14, 6332-6342.

38 Petersen, J.; Møller, K.B.; Rey, R.; Hynes, J.T. Ultrafast Librational Relaxation of $\mathrm{H}_{2} \mathrm{O}$ in Liquid Water. J. Phys. 
Chem. B 2013, 117, 4541-4552.

${ }^{39} \mathrm{Li}, \mathrm{T}$. Efficient Criterion To Evaluate Linear Response Theory in Optical Transitions J. Chem. Theory Comput., 2017, 13, 1867-1873.

${ }^{40}$ Heid, E.; Moser, W.; Schröder, C. On the validity of linear response approximations regarding the solvation dynamics of polyatomic solutes. Phys.Chem.Chem.Phys. 2017, 19, 10940-10950.

41 Schile, A.J.; Thompson, W.H. Tests for, origins of, and corrections to non-Gaussian statistics. The dipole-flip model. J. Chem. Phys. 2017, 146, 154109.

${ }^{42}$ Heid, E.; Schröder, C. Effect of a Tertiary Butyl Group on Polar Solvation Dynamics in Aqueous Solution: A Computational Approach. J. Phys. Chem. B 2017, 121, 96399646.

43 Gerecke, M.; Richter, C.; Quick, M.; Ioffe, I.N.; Mahrwald, R.; Kovelenko, S.A.; Ernsting, N.P. Effect of a Tertiary Butyl Group on Polar Solvation Dynamics in Aqueous Solution: Femtosecond Fluorescence Spectroscopy. J. Phys. Chem. B, 2017, 121, 9631-9638.

44 Videla, P.E.; Rossky, P.J.; Laria, D. A quantum molecular dynamics study of aqueous solvation dynamics. J. Chem. Phys. 2013, 139, 164506.

45 Laird, B.B.; Thompson, W.H. On the connection between Gaussian statistics and excited-state linear response for time-dependent fluorescence. J. Chem. Phys., 2007, 126, 211104.

46 Bedard-Hearn, M.J.; Larsen, R.E.; Schwartz, B.J. Understanding Nonequilibrium Solute and Solvent Motions through Molecular Projections: Computer Simulations of Solvation Dynamics in Liquid Tetrahydrofuran (THF). $J$. Phys. Chem. B 2003, 107, 14464-14475.

47 Sajadi, M.; Ernsting, N.P. Excess Dynamic Stokes Shift of Molecular Probes in Solution. J. Phys. Chem. B 2013 , $117,7675-7684$.

${ }^{48}$ Konart, A.; Loxovoy, V.V.; Dantus, M. Solvation StokesShift Dynamics Studied by Chirped Femtosecond Laser Pulses. J. Phys. Chem. Lett. 2012, 3, 2458-2464.

49 Joutsuka, T.; Thompson, W.H.; Laage, D. Vibrational Quantum Decoherence in Liquid Water. J. Phys. Chem. Lett. 2016, $7,616-621$.

50 Silori, Y.; Seliya, P.; De, A.K. Early Time Solvation Dynamics Probed by Spectrally Resolved Degenerate PumpProbe Spectroscopy. ChemPhysChem 2019, 20, 14881496.

51 Heid, E.; Schmode, S.; Chatterjee, P.; MacKerell, A.D.; Shröder, C. Solvation dynamics: improved reproduction of the time-dependent Stokes shift with polarizable empirical force field chromophore models. Phys.Chem.Chem.Phys. 2019, 21, 17703-17710.

52 Sun, X. Hybrid equilibrium-nonequilibrium molecular dynamics approach for two-dimensional solutepump/solvent-probe spectroscopy. J. Chem. Phys. 2019, 151, 194507.

53 Messina, F.; Bräm, O.; Cannizzo, A.; Cherqui, M. Realtime observation of the charge transfer to solvent dynamics. Nat. Comm. 2013, 4, 2119.

${ }^{54}$ Koneshan, S.; Rasaiah, J.C.; Lynden-Bell, R.M.; Lee, S.H. Solvent Structure, Dynamics, and Ion Mobility in Aqueous Solutions at 25 C. J. Phys. Chem. B 1998, 102, 4193-4204.

55 Rasaiah, J.C.; Lynden-Bell, R.M. Computer Simulation Studies of the Structure and Dynamics of Ions and NonPolar Solutes in Water. Phil. Trans. R. Soc. Lond. A
2001, 359, 1545-1574.

56 Re, M.; Laria, D. Dynamics of Solvation in Supercritical Water. J. Phys. Chem. B 1997, 101, 10494-10505.

57 Biswas, R.; Bagchi, B., Ion Solvation Dynamics in Supercritical Water. Chem. Phys. Lett. 1998, 290, 223-228.

58 Duan, J.; Shim, Y.; Kim, H.J. Solvation in Supercritical Water. J. Chem. Phys. 2006, 124, 204504.

59 Zhang, X.X.; Liang, M.; Ernsting, N.P.; Maroncelli, M. Conductivity and Solvation Dynamics in Ionic Liquids. $J$. Phys. Chem. Lett. 2013, 4, 1205-1210.

60 Terranova, Z.L.; Corcelli, S.A. On the Mechanism of Solvation Dynamics in Imidazolium-Based Ionic Liquids. $J$. Phys. Chem. B 2013, 117, 15659-15666.

61 Terranova, Z.L.; Corcelli, S.A. Decompositions of Solvent Response Functions in Ionic Liquids: A Direct Comparison of Equilibrium and Nonequilibrium Methodologies. $J$. Phys. Chem. B 2018, 122, 6823-6828.

62 Changenet-Barret, P.; Choma, C.T.; Gooding, E.F.; DeGrado, W.F.; Hochstrasser, R.M. Ultrafast dielectric response of proteins from dynamics Stokes shifting of coumarin in calmodulin. J. Phys. Chem. B 2000 104, 9322-9329.

63 Pal, S.K.; Peon, J.; Zewail, A.H. Biological water at the protein surface: Dynamical solvation probed directly with femtosecond resolution. PNAS 2002, 99, 1763-1768.

${ }^{64}$ Golosov, A.A.; Karplus, M. Probing Polar Solvation Dynamics in Proteins: A Molecular Dynamics Simulation Analysis. J. Phys. Chem. B 2007, 111, 1482-1490.

65 Abbyad, P.; Childs, W.; Shi, X.; Boxer, S.G. Dynamic Stokes shift in green fluorescent protein variants. PNAS 2007, 104 20189-20194.

66 Rodríguez, J.; Martí, J.; Guàrdia, E.; Laria, D. Exploring the Picosecond Time Domain of the Solvation Dynamics of Coumarin 153 within beta-Cyclodextrins. J. Phys. Chem. B 2008, 112, 8990-8998.

67 Halle, B.; Nilsson, L. Does the Dynamic Stokes Shift Report on Slow Protein Hydration Dynamics? J. Phys. Chem. B 2009, 113, 8210-8213.

${ }^{68}$ Chang, C.W.; Guo, L.; Kao, Y.T.; Li, J.; Tan, C.; Li, T.; Saxena, C.; Liu, Z.; Wang, L.; Sancar, A. et al. Ultrafast solvation dynamics at binding and active sites of photolyases. PNAS 2010, 107, 2914-2919.

69 Qin, Y.; Wang, L.; Zhong, D. Dynamics and mechanism of ultrafast water-protein interactions. PNAS 2016, 113 8424-8429.

70 Jumper, C.C.; Arpin, P.C.; Turner, D.B.; McClure, S.D.; Rafig, S.; Dean, J.C.; Cina, J.A.; Kovac, P.A.; Mirkovic, T.; Scholes, G.D. Broad-Band Pump-Probe Spectroscopy Quantifies Ultrafast Solvation Dynamics of Proteins and Molecules. J. Phys. Chem. Lett. 2016, 7, 4722-4731.

71 Laage, D.; Elsaesser, T.; Hynes, J.T. Water Dynamics in the Hydration Shells of Biomolecules. Chem. Rev. 2017, 117, 10694-10725.

72 Liu, Y.; Guchhait, B.; Siebert, T.; Fingerhut, B.P.; Elsaesser, T. Molecular couplings and energy exchange between DNA and water mapped by femtosecond infrared spectroscopy of backbone vibrations. Struct. Dyn. 2017, 4, 044015.

73 Sardana, D.; Yadav, K.; Shweta, H.; Clovis, N.S.; Alam, P.; Sen, S. Origin of Slow Solvation Dynamics in DNA: DAPI in Minor Groove of Dickerson-Drew DNA.J. Phys. Chem. B 2019, 123, 10202-10216.

${ }^{74}$ Honegger, P.; Heid, E.; Schmode, S.; Schröder, C.; Steinhauser, O. Changes in protein hydration dynamics by en- 
capsulation or crowding of ubiquitin: strong correlation between time-dependent Stokes shift and intermolecular nuclear Overhauser effect. RSC Adv. 2019, 63, 3698236993.

75 Heid, E.; Braun, D. Fundamental limitations of the timedependent Stokes shift for investigating protein hydration dynamics. Phys. Chem. Chem. Phys. 2019, 21, 44354443.

${ }^{76}$ Levi, G.; Papai, M.; Henriksen, N.E.; Dohn, A.O.; Møller, K.B. Solution Structure and Ultrafast Vibrational Relaxation of the PtPOP Complex Revealed by Delta SCFQM/MM Direct Dynamics Simulations. J. Phys. Chem. C 2018, 122, 7100-7119.

77 Prampolini, G.; Ingrosso, F.; Segalina, A.; Caramori, S.; Foggi, P.; Pastore, M. Dynamical and Environmental Effects on the Optical Properties of an Heteroleptic Ru(II)Polypyridine Complex: A Multilevel Approach Combining Accurate Ground and Excited State QM-Derived Force Fields, MD and TD-DFT. J. Chem. Theor. Comp. 2019, 15, 529-545.

78 Remsing, R.C.; Klein, M.L. Solvation dynamics in water confined within layered manganese dioxide. Chem. Phys. Lett. 2017, 683, 478-482.

${ }^{79}$ Calero, C.; Sibert, E.L.; Rey, R. Self-thermophoresis at the nanoscale using light induced solvation dynamics. Nanoscale, 2020, 12, 7557-7562.

80 Tran, V.; Schwartz, B.J. Role of Nonpolar Forces in Aqueous Solvation: Computer Simulation Study of Solvation Dynamics in Water Following Changes in Solute Size, Shape and Charge. J. Phys. Chem. B 1999, 103, 55705580 .

81 Aherne, D.; Tran, V.; Schwartz, B.J. Nonlinear, Nonpolar Solvation Dynamics in Water: the Roles of Electrostiction and Solvent Translation in the Breakdown of Linear Response. J. Phys. Chem. B 2000, 104, 5382-5394.

${ }^{2}$ Grote, R.F.; Hynes, J.T. The Stable States Picture of Chemical Reactions.II. Rate Constants for Condensed and Gas Phase Reaction Models. J. Chem. Phys. 1980, 73,2715-2732.

${ }^{83}$ Bergsma, J.P.; Gertner, B.J.; Wilson, K.R.; Hynes, J.T. Molecular Dynamics of a Model SN2 Reaction in Water. J. Chem. Phys., 1987, 86, 1356-1376.

84 Burghardt, I.; Laage, D.; Hynes, J.T. On the Dissociation of Aromatic Radical Anions in Solution. II. Reaction Path and Rate Constant Analysis. J. Phys. Chem. A 2003, 10\%, 11292-11306.

85 Malhado, J.; Spezia, R.; Hynes, J.T. Dynamical Friction Effects on the Photoisomerization of a Model Protonated Schiff Base in Solution. J. Phys. Chem. A 2011, 115, 3720-3735.

86 Ruiz-Pernia, J.; Tuñón, I.; Moliner, V.; Hynes, J.T. Dynamic Effects on Reaction Rates in a Michael Addition Catalyzed by Chalcone Isomerase. Beyond the Frozen Environment Approach. J. Amer. Chem. Soc. 2008, 130, 7477-7488.

87 Staib, A.; Hynes, J.T.; Borgis, D. Proton Transfer in Hydrogen-Bonded Acid-Base Complexes in Polar Solvents. J. Chem. Phys. 1995, 102, 2487-2505.

88 Weaver, M.J.; McManis, G.E.; Wlodzimiez, J.; Barbara, P.F. Importance of fast solvent relaxation components to electron-transfer rates: comparisons between barriercrossing frequencies and subpicosecond time-resolved solvation dynamics. J. Phys. Chem. 1990, 94, 1715-1719.

${ }^{89}$ Ciccotti, G.; Ferrario, M.; Hynes, J.T.; Kapral, R. Dy- namics of ion pair interconversion in a polar solvent. $J$. Chem. Phys. 1990, 93, 7137-7147.

${ }^{90}$ Ladanyi, B.M.; Stratt, R.M. Short-Time Dynamics of Solvation: Relationship between Polar and Nonpolar Solvation. J. Phys. Chem. 1996, 100, 1266-1282.

${ }^{91}$ Castner, E.W.; Maroncelli, M. Solvent dynamics derived from optical Kerr effect, dielectric dispersion, and timeresolved stokes shift measurements: an empirical comparison. J. Mol. Liq. 1998, 77, 1-36.

92 Reid, P.J.; Barbara, P.F. Dynamic Solvent Effect on Betaine-30 Electron-Transfer Kinetics in Alcohols. $J$. Phys. Chem. 1995, 99, 3554-3565.

$93 \mathrm{Su}, \mathrm{S}$.; Simon, J.D. Importance of molecular size on the dynamics of solvent relaxation. J. Phys. Chem. 1989 93, 753-758.

${ }^{94}$ Hynes, J.T. In Ultrafast Spectroscopy; Simon, J., Ed.; Kluwer: Dordrecht, 1994; pp 345-381.

95 Madden, P.A.; Kivelson, D. A Consistent Molecular Treatment of Dielectric Phenomena. Adv. Chem. Phys. 1984, LVI, 467-566.

96 Neria, E.; Nitzan, A. Numerical simulations of solvation dynamics in electrolyte solutions. J. Chem. Phys. 1994, 100, 3855-3868.

97 Martins, L.R.; Skaf, M.S. Computer simulations of the solvation dynamics of Coumarin 153 in dimethylsulfoxide. Chem. Phys. Lett. 2003, 370, 683-689.

98 Berendsen, H. J. C.; Grigera, J.R.; Straatsma, T.P. The Missing Term in Effective Pair Potentials. J. Phys. Chem 1987, 91, 6269-6271.

99 Toukan, K.; Rahman, A. Molecular Dynamics Study of Atomic Motions in Water. Phys. Rev. B 1985, 31, 26432648.

100 Berendsen, H.J.C.; Postma, J.P.M.; van Gunsteren, W.F.; DiNola, A.; Haak, J.R. Molecular Dynamics with Coupling to an External Bath. J. Chem. Phys. 1984, 81, 3684-3690.

101 Walrafen,G. E. Weak Raman bands from water. J. Chem. Phys. 1973, 59, 2646-2650.

102 Walrafen, G. E. Raman Spectral Studies of Water Structure. J. Chem. Phys. 1964, 40, 3249-3256.

103 Huse, N.; Ashihara, S.; Nibbering, E. T. J.; Elsaesser, T. Ultrafast vibrational relaxation of $\mathrm{O}-\mathrm{H}$ bending and librational excitations in liquid $\mathrm{H} 2 \mathrm{O}$. Chem. Phys. Lett. 2005, 404, 389-393.

104 Ashihara, S.; Huse, N.; Espagne, A.; Nibbering, E. T. J.; Elsaesser, T. Ultrafast structural dynamics of water induced by dissipation of vibrational energy. J. Phys. Chem. A 2007, 115, 743-746.

105 Pakoulev, A.; Wang, Z.; Pang, Y.; Dlott, D. D. Vibrational energy relaxation pathways of water. Chem. Phys. Lett. 2003, 380, 404-410.

106 One might also consider an equilibrium TCF based exclusively on an excited state, i.e. for both the distribution and dynamics; while this version - which is the result of an alternate and much less common "linear response" approximation ${ }^{13}$ - turns out to be a description superior to that of the ground state $\mathrm{TCF}^{13-15,45}$ it is the latter that is by far predominantly employed in the literature.

107 Some analytic models also give an initial Gaussian behaviour, ${ }^{25,27}$ but with inappropriate frequencies. ${ }^{15,25}$

108 In some intermediate stage of manipulations of the quadratic term, one can encounter the total excited state force $\boldsymbol{F}_{e}=\boldsymbol{F}_{g}+\boldsymbol{F}_{c}$ occurring in the average $\left\langle\boldsymbol{F}_{e} \cdot \boldsymbol{F}_{c}\right\rangle_{g}$ rather than only the Coulomb force $\boldsymbol{F}_{c}$ which we always 
emphasize in connection with non-inertial character. For example, as shown in SI Sec. S4, this arises from the noninertial contribution to the Liouville operator Eq. 3. However, the force $\boldsymbol{F}_{g}$ in the average vanishes, as shown in SI Sec. S4.

109 The demonstration of this exact equivalence in the solvation dynamics context is novel. Also in the solvation dynamics context, we note that for different solutes and solvents, other close numerical similarities have been noted: of the initial Gaussian behavior of the ground state TCF to the short time behavior of the related nonequilibrium FSF $S(t)^{13-15}$ and of the initial Gaussian behavior of a fully excited state TCF (excited state initial distribution and dynamics) and the short time behavior of the related nonequilibrium FSF $S(t) .{ }^{13}$ However, our equivalence result is completely consistent with the free energy-based demonstration that the ground and excited state free energy curves' curvatures in the solvent coordinate are identical, given in Tachiya, M. Relation between the electrontransfer rate and the free energy change of reaction. $J$. Phys. Chem., 1989, 93, 7050-7052. (Note that the equivalence discussed here is different from the exact equivalence of the two different (non-inertial and inertial perspective) quadratic forms in the nonequilibrium results Eqs. 7 and 8.)

110 However, the work/power orientational calculations of Sec. III B show that strong Coulombic torques driving non-inertial motion are in fact operative in this time regime, confirming the suggestions given in Sec. III A.

111 For example, alternate examination of the spatial and orientational fates of trajectories ${ }^{13,14}$ or comparison with orientational correlation functions ${ }^{14}$ can be effected. In our view, the value of such investigations is far greater for the nonequilibrium case than for reference equilibrium TCFs.

112 Wilkins, D.M.; Manolopoulos, D.E.; Pipolo, S.; Laage, D.; Hynes, J.T. Nuclear Quantum Effects in Water Reorientation and Hydrogen-Bond Dynamics. J. Phys. Chem. Lett. 2017, 8, 2602-2607.

113 Sibert III, E.L.; Reinhardt, W.P.; Hynes, J.T. Intramolecular Vibrational Relaxation and Spectra of $\mathrm{CH}$ and $\mathrm{CD}$ Overtones in Benzene and Perdeuterobenzene. J. Chem. Phys. 1984,81, 1115-1134.

114 Sibert III, E.L.; Hynes, J.T.; Reinhardt, W.P. Classical Dynamics of Highly Excited $\mathrm{CH}$ and CD Overtones in Benzene and Perdeuterobenzene. J. Chem. Phys. 1984, 81, 1135-1144.

115 Hybl, J.D.; Christophe, Y.; Jonas, D.M. Peak shapes in femtosecond 2D correlation spectroscopy. Chem. Phys. 2001, 266, 295-309, and later solvation dynamics papers from the Jonas group. 
TOC graphic

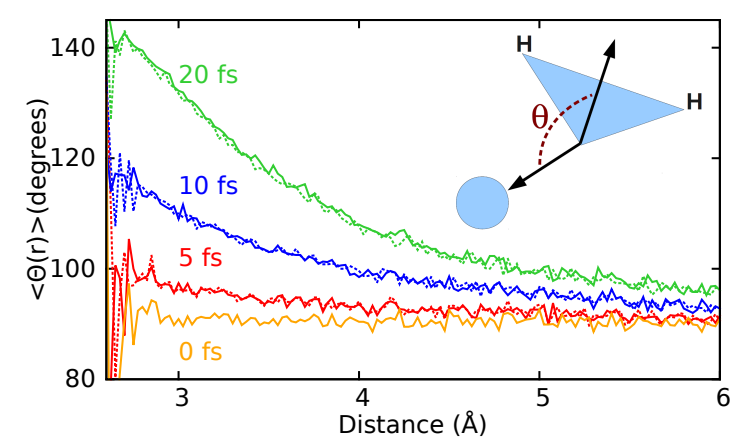

